\title{
Study of the Impact Energy Releasing Characteristics of Al/PTFE/W Energetic Jets
}

\author{
Fuhai Li, ${ }^{1}$ Hantao Liu $\mathbb{D}^{1}{ }^{1}$ and Yanwen Xiao ${ }^{2}$ \\ ${ }^{1}$ College of Mechatronic Engineering, North University of China, Taiyuan 030051, China \\ ${ }^{2}$ Unit 32381, PLA, Beijing 100071, China \\ Correspondence should be addressed to Hantao Liu; 20050052@nuc.edu.cn
}

Received 15 January 2020; Revised 14 May 2020; Accepted 19 August 2020; Published 30 August 2020

Academic Editor: Zhaoye Qin

Copyright $(2020$ Fuhai Li et al. This is an open access article distributed under the Creative Commons Attribution License, which permits unrestricted use, distribution, and reproduction in any medium, provided the original work is properly cited.

Compared with traditional jets, energetic jets have more efficient damage effects. To study the reaction characteristics of polytetrafluoroethylene- (PTFE-) based energetic jets under impact loading, the static mechanical properties of Al/PTFE/W composite energetic materials are studied by using a universal testing machine at a strain rate of $0.01 \mathrm{~s}^{-1}$, and the dynamic mechanical properties are tested on a slip Hopkinson pressure bar (SHPB) system at a strain rate of 1000 5500 s ${ }^{-1}$. A dynamic energy acquisition system is established to quantify the energy generated by the response of the Al/PTFE/W energetic jets to impact targets. The effects of the material proportion and impact energy on the mechanical and energy release properties of the Al/ PTFE/W energetic jets are analyzed. The results show that the Al/PTFE/W composite has an obvious strain rate effect. As the $W$ content in the composite increases, the yield strength and compressive strength of the material increase gradually, but the strain at break decreases. When the $W$ content is $45 \%$, the peak pressure, total release energy, pressure platform duration, and total pressure duration of the Al/PTFE/W energetic jets are the highest. As the impact energy increases, the pressure peak and energy release values of the energetic jets increase. At an impact energy threshold of $106.1 \mathrm{MJ} / \mathrm{m}^{2}$, the chemical reaction of the Al/PTFE/ $\mathrm{W}(45 \%)$ energetic jets is saturated. The results provide a theoretical and experimental basis for the application of energetic jets.

\section{Introduction}

Metal/fluoropolymer composites are formed when active metal powders are uniformly mixed into a fluoropolymer matrix, are cold pressed, and undergo a sintering hardening process to enhance their mechanical strength [1]. These composites are inert under normal conditions and insensitive to friction, heat, fusing, and detonation. However, the extreme forces upon impact cause the components to react with each other and release more energy than highly explosive components [2]. This kind of material can be prepared into next-generation ammunition by replacing components normally made of inert materials, such as fragmentation warheads, shaped-charge warheads, penetrating warheads, and projectiles and can cause additional damage to the target through additional chemical reactions.

In recent years, researchers have made notable progress on shock-induced chemical reactions of metal/fluoropolymer composites. Feng et al. [3] found that the sintering temperature controls the crystallinity of the PTFE matrix, and the reactivity and toughness increase as the crystallinity decreases. Ames [4] and Mock and Drotar [5] studied the shock initiation of rods through Taylor impact tests and established the relationship between the ignition delay time and the impact energy. Shuai et al. [6], Xie et al. [7], and Xin et al. [8] conducted an experimental study on the penetration of PTFE$\mathrm{Al}$ reactive fragments into steel targets, the damage of diesel fuel tanks, and the application of an active liner and determined that metal/fluoropolymer composites can achieve greater damage efficiency than conventional materials. Wang et al. [9, 10], Zhang et al. [11], and Luo et al. [12] used a vented chamber to measure the released energy of PTFE-Al energetic fragments under various impact conditions and concluded that the extent of the reaction was influenced by the impact velocity, strength properties, and target thickness.

However, compared to conventional metals and alloys, active metal/fluoropolymer composites, such as PTFE/Al, have relatively low densities and material strength 
properties. Therefore, $W$ particles are typically added to the composites to increase the density and structural strength. Recently, researchers have carried out studies on the reaction characteristics of active metal/fluoropolymer composites enhanced by $W$ particles. Wang et al. [13] studied the effect of the $W$ content on the reaction energy of W-PTFE-Al composites in both oxygen and argon and determined that the insensitivity to impact loading increased as the $W$ mass ratio increased. Zhang et al. [14] researched the effects of the particle size of $\mathrm{Al}$ and $W$ on the combustion characteristics and dynamic response of $\mathrm{W}$-PTFE-Al composites and indicated that fine aluminum and coarse $W$ contributed to an increase in the reaction energy. The aforementioned results show that the strength and reactivity of composites can be enhanced by properly adding W.

In the above article, the energy release characteristics of energetic materials under impact loading were studied in the form of specimens or fragments, but few studies have focused on applications of the energetic materials of jets. After all, the formation process of the fragment and the jet and the mechanism of releasing the energy are completely different. Therefore, it is necessary to study the energy release characteristics of energetic materials in jet form. Because of the high loading strain rate, it is especially important to test the dynamic response of energetic jets under dynamic loading. The slip Hopkinson pressure bar (SHPB) experimental technology has become an important experimental method to study the dynamic response of materials under impact loading because of its advantages such as a simple structure, convenient operation, exquisite measurement method, and easily controllable waveform [15]. The dynamic mechanical properties of $\mathrm{Al} / \mathrm{PTFE} / \mathrm{W}$ composite energetic materials with $W$ contents of $0 \%, 25 \%, 45 \%$, and $60 \%$ are studied herein by using the SHPB system. A dynamic energy acquisition system is established to quantify the energy released by the response of $\mathrm{Al} / \mathrm{PTFE} / \mathrm{W}$ energetic jets to impact targets. The effects of the material ratio and impact energy on the mechanical and impact energy properties of the Al/PTFE/W energetic jets are analyzed.

\section{Materials and Methods}

2.1. Material Preparation. The equation for the reaction of $\mathrm{Al}$ with PTFE is $4 \mathrm{Al}+3 \mathrm{C}_{2} \mathrm{~F}_{4}=4 \mathrm{AlF}_{3}+6 \mathrm{C}$. To fully carry out the chemical reaction between $\mathrm{Al}$ and $\mathrm{PTFE}$, the mass ratio of Al to PTFE should satisfy the ratio of the zero-oxygen balance equation, which is $9: 25$. $W$ does not participate in the chemical reaction between $\mathrm{Al}$ and PTFE.

Ball mill mixing of the $\mathrm{Al}, W$, and PTFE powders was performed using a ball mill (QM-35P4, Changsha Tianchuang Instrument Factory) with high-purity aluminum ceramic balls with diameters of 1 and $3 \mathrm{~mm}$ and a ball-topowder mass ratio of $10: 1$. The particle sizes of the $\mathrm{Al}, W$, and PTFE powders (Beijing Xing Rong Yuan Technology Co., Ltd.) were $5 \mu \mathrm{m}, 5 \mu \mathrm{m}$, and $25 \mu \mathrm{m}$, respectively. The rotation speed was $200 \mathrm{r} / \mathrm{min}$, the ball milling time was $480 \mathrm{~min}$, and the milling was followed by drying in a vacuum oven. The dried powder was evenly loaded into a graphite mold and placed in a custom hot-pressing sintering furnace
(R-C-ZKQY-07, Chenrong Electric Furnace Co., Ltd., Shanghai, China) in an Ar gas atmosphere, as shown in Figure 1 . The heating rate was set as $1^{\circ} \mathrm{C} / \mathrm{min}$ until $380^{\circ} \mathrm{C}$ was reached, and the sintering temperature was maintained for 6 hours. Pressurization started $10 \mathrm{~min}$ before reaching a sintering temperature of $380^{\circ} \mathrm{C}$, and the applied pressure was $30 \mathrm{MPa}$. Then, the temperature was lowered at a rate of $30^{\circ} \mathrm{C} /$ $\mathrm{h}$ until $315^{\circ} \mathrm{C}$ was reached and maintained for 4 hours. Finally, the temperature was lowered at a rate of $30^{\circ} \mathrm{C} / \mathrm{h}$ until room temperature was reached. According to the above process, $\mathrm{Al} / \mathrm{PTFE} / \mathrm{W}$ energetic materials with $W$ masses of $0 \%, 25 \%, 45 \%$, and $60 \%$ were prepared. The energetic material ratio and density are shown in Table 1 . The preparation process of the Al/PTFE/W composites is shown in Figure 2.

2.2. Experimental Methods. The quasistatic compression mechanical properties of the Al/PTFE/W samples with different $W$ mass ratios were tested by a universal testing machine at a strain rate of $0.01 \mathrm{~s}^{-1}$. The dimensions of the compression samples were $4 \times 4 \times 10 \mathrm{~mm}$. Each sample was tested at least five times, and the test data from three measurements with good agreement were averaged.

The dynamic compressive mechanical properties of the Al/PTFE/W samples were tested on an SHPB system (Key Laboratory of Science and Technology on Materials under Shock and Impact, Beijing, China) at a strain rate of $1000 \sim 5500 \mathrm{~s}^{-1}$. The experimental system consisted of a gas gun, a striker bar, an incident bar, a transmission bar, an absorption bar, and a data acquisition system. Different strain rates were obtained by changing the atmospheric pressure applied to the impact rod. When the stress pulse propagates to the interface between the incident bar and the test piece, it will cause high-speed deformation of the test piece and form a reflection wave that propagates in the incident bar and a transmission wave that propagates in the transmission bar. At the same time, the strain gauge pasted on the incident bar and transmission bar transmits the measured incident signal, reflected signal, and transmission signal to the dynamic strain gauge for strain amplification, and then through the signal acquisition of the digital oscilloscope and data processing of the computer, the interrelation among the internal stress, strain, strain rate, and time of the test piece can be obtained, as shown in Figures 3 and 4. It is necessary to apply a small amount of vacuum grease on both ends of the test piece as a lubricant to reduce the influence of the friction effect on the experiment.

The Al/PTFE/W composite has low mechanical strength and mechanical impedance, and the propagation speed of the stress wave is slow. According to the assumption of uniformity, the stress wave should be transferred more than three times in the specimen to be considered as dynamic equilibrium, and the experimental results can be considered as reliable. However, the SHPB experimental loading wave is a strong discontinuity wave, and the sudden loading of the specimen will cause the stress at one end of the stress to be significantly greater than that at the other end, so it is difficult to ensure the uniformity of the internal stress and deformation of the specimen, and the error of the 


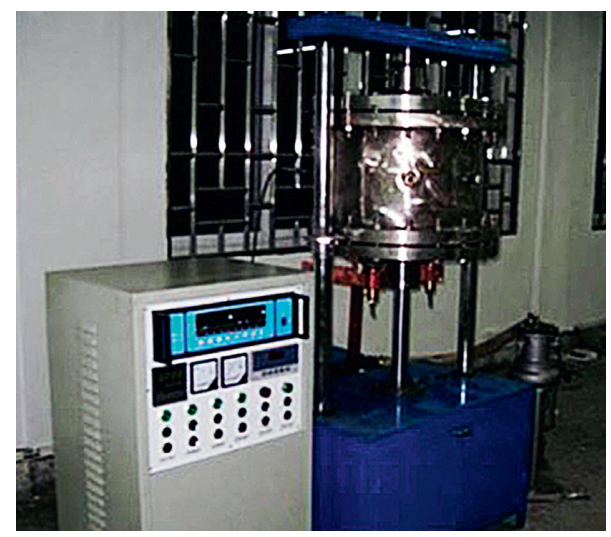

Figure 1: Hot-pressing sintering furnace.

TABle 1: Energetic material ratios and densities.

\begin{tabular}{lccc}
\hline Number & Mass ratio \% (W/Al/PTFE) & Theoretical density $\left(\mathrm{kg} / \mathrm{m}^{3}\right)$ & Actual density $\left(\mathrm{kg} / \mathrm{m}^{3}\right)$ \\
\hline 1 & $0 / 26.5 / 73.5$ & 2.31 & 2.29 \\
2 & $25 / 19.9 / 55.1$ & 2.97 & 2.89 \\
3 & $45 / 14.6 / 40.4$ & 3.83 & 3.75 \\
4 & $60 / 10.6 / 29.4$ & 4.90 & 4.81 \\
\hline
\end{tabular}

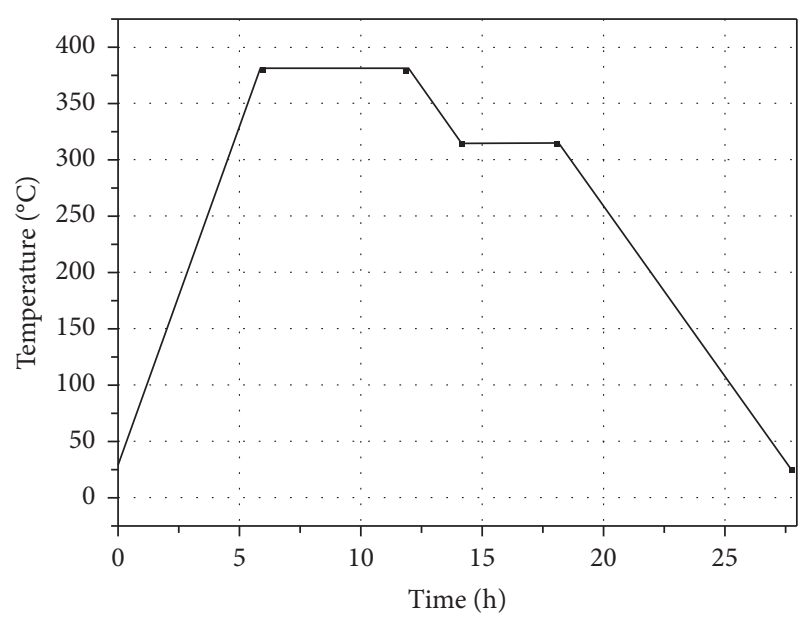

Figure 2: Process used to prepare the Al/PTFE/W composites.

experimental data is large. To make the internal stress of the specimen uniform, a waveshaper is added in the front of the incident bar to extend the rise time of the load, that is, to slow down the rising edge of the incident wave.

The rod was made of steel with an elastic modulus of $200 \mathrm{GPa}$ and a diameter of $14.5 \mathrm{~mm}$. The bullet had a diameter of $14.5 \mathrm{~mm}$ and a length of $20 \mathrm{~cm}$. The size of the samples was $10 \times 5 \mathrm{~mm}$. The schematic diagram of the SHPB test system is shown in Figure 5. The experimental setup of SHPB is shown in Figure 6. The dynamic compression sample is shown in Figure 7.

A dynamic energy acquisition system was constructed to study the impact energy release characteristics of the $\mathrm{Al} /$ PTFE/W energetic jets, as shown in Figures 8 and 9. The dynamic energy acquisition system consisted of an Al/PTFE/ $\mathrm{W}$ composite liner (with a hemispherical shape, a diameter

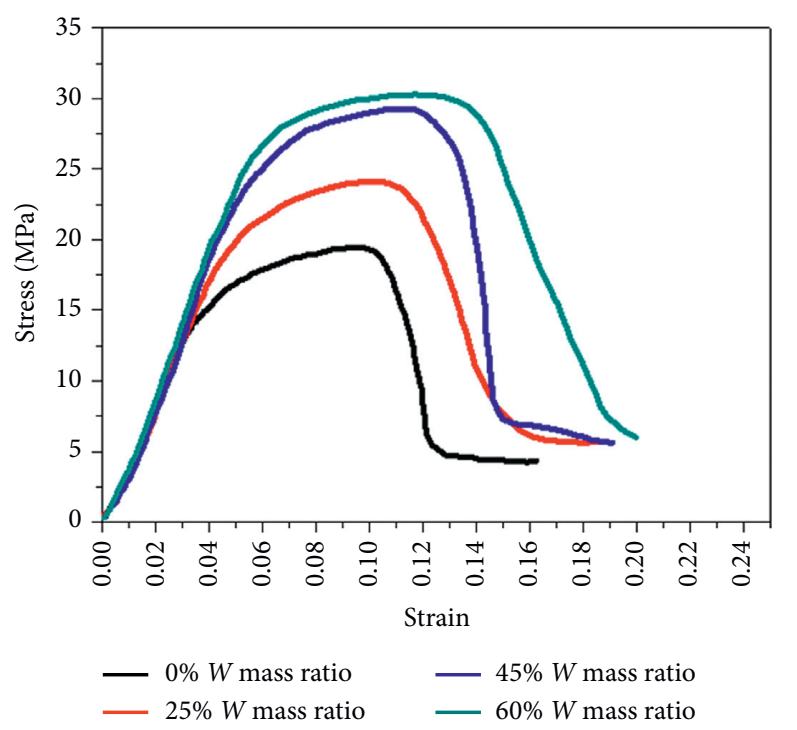

FIGURE 3: Quasistatic compressive stress-strain curves of the $\mathrm{Al} /$ $\mathrm{PTFE} / \mathrm{W}$ energetic materials.

of $40 \mathrm{~mm}$, an equal wall thickness of $2 \mathrm{~mm}$, and an equivalent cone angle of $120^{\circ}$, as shown in Figure 10), a charge (poly- 8 with a density of $1787 \mathrm{~kg} / \mathrm{m}^{3}$ and a detonation velocity of $8390 \mathrm{~m} / \mathrm{s}$ ), a steel test container (with a diameter of $380 \mathrm{~mm}$ and a volume of $14.67 \mathrm{~L}$ ), a pressure testing system (a BZ2202 multichannel dynamic strain gauge and a TST3125 dynamic test analyzer), a pressure sensor (with a $-20 \sim 20 \mathrm{kN}$ pressure range, a sampling rate of $20 \mathrm{kHz}$, a sampling length of $58 \mathrm{~s}$, a delay of $2 \mathrm{~ms}$, and a control level of $0.15 \mathrm{~V})$, a conductor, front and rear sealing plates, several Q235 steel target plates with different thicknesses, a steel protective plate, a high-speed camera, and support. The 


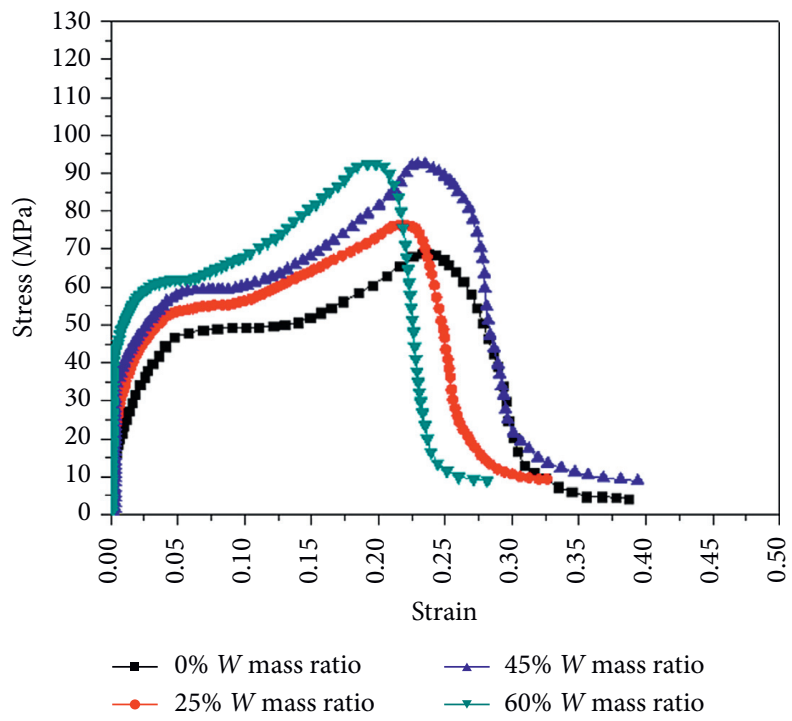

Figure 4: Stress-strain curves of Al/PTFE/W energetic materials with different $W$ contents (the strain rate was $4500 \mathrm{~s}^{-1}$ ).

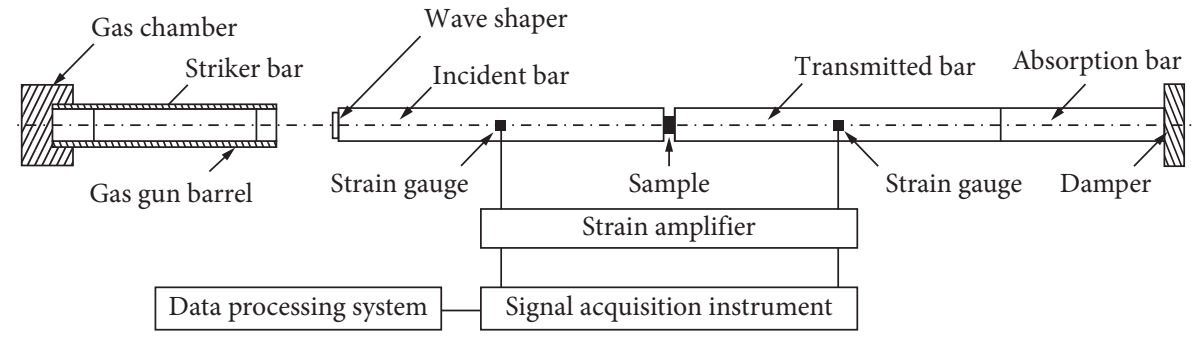

Figure 5: Schematic diagram of the SHPB test system.

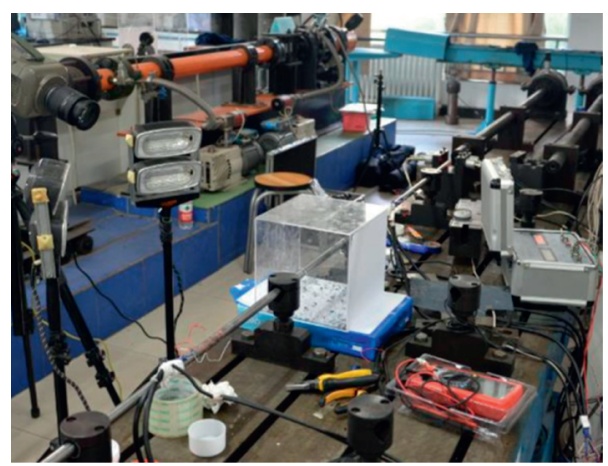

Figure 6: Experimental setup of SHPB.

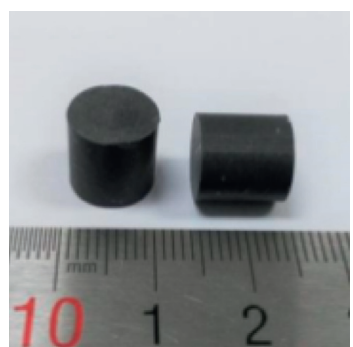

(a)

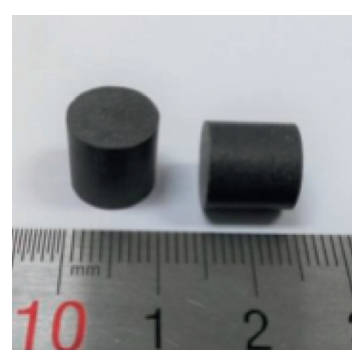

(b)

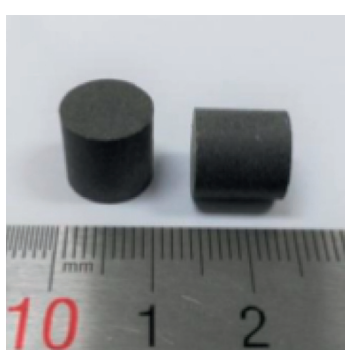

(c)

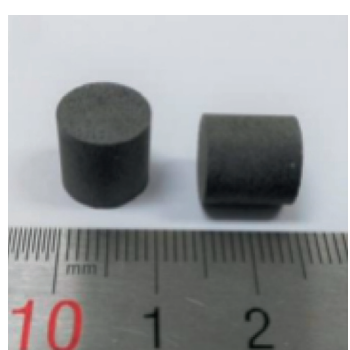

(d)

Figure 7: Dynamic compression samples: (a) $0 \%$ W. (b) $25 \%$ W. (c) $45 \%$ W. (d) $60 \%$ W. 


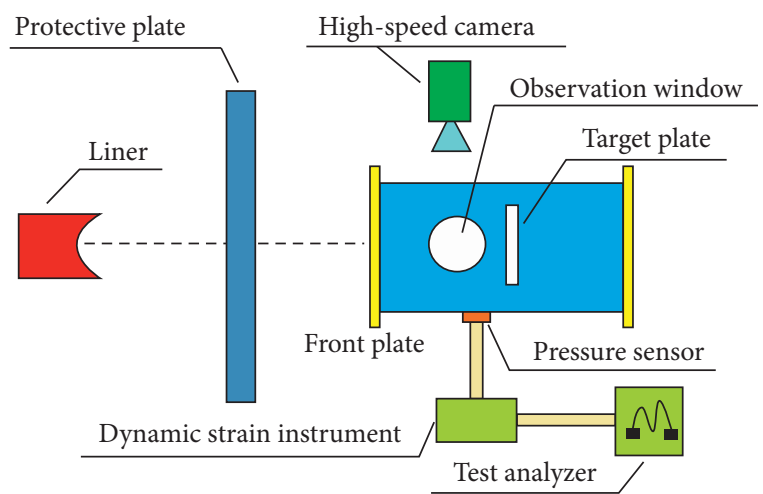

FIgURE 8: Schematic diagram of the energy acquisition system.

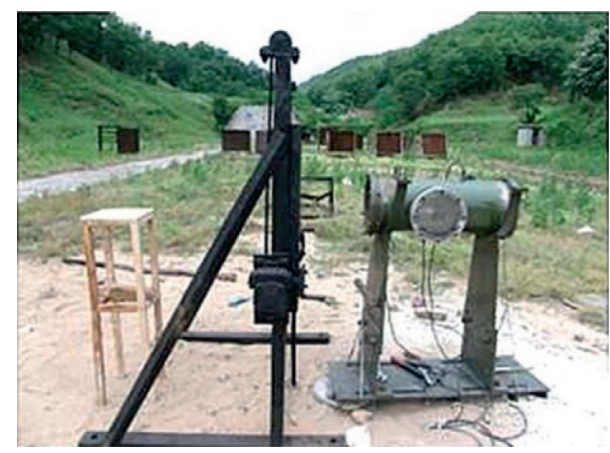

Figure 9: Experimental device in the field.

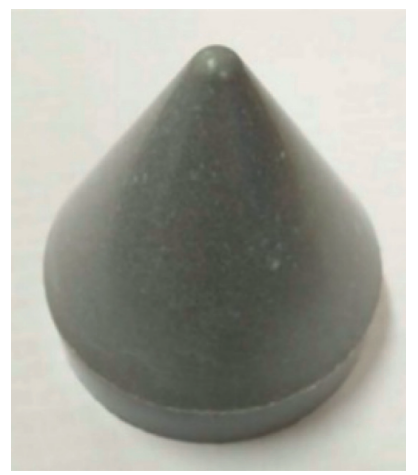

FIgUre 10: Al/PTFE/W composite liner.

target plate was fixed to the steel support frame inside the test container by bolts, and a protective plate was placed between the test container and the liner to block detonation products and minimize the impact on the test system. To ensure that the jet entered the test container smoothly, an $80 \mathrm{~mm}$ pressure relief hole was located in the center of the protective plate and the front sealing plate. The axis of the liner was calibrated by a laser, and the heights of the protective plate and the front sealing plate were adjusted to be the same as that of the axis of the liner. The liner was placed on the launcher and was driven by explosive action to form a jet, which passed through the preperforated protective plate and impacted the steel baffle to produce a violent chemical reaction, releasing a large amount of heat and causing the air in the chamber to expand into an overpressure condition. The sensor at the front of the test container recorded the voltage-time signal due to the energy released by the energetic material in the jet, and the original signal was smoothed after filtering. The energy release pressure-time curve was obtained by numerical calculation.

Two series of experiments were designed to investigate the effects of the ratio of the energetic materials and impact conditions on the impact energy release characteristics of the $\mathrm{Al} / \mathrm{PTFE} / \mathrm{W}$ energetic jets.

In one set of experiments, the effect of different energetic material ratios on the energy release was investigated. The thickness of the steel diaphragm was $4 \mathrm{~mm}$, and the mass ratio of $W$ was $0 \%, 25 \%, 45 \%$, and $65 \%$.

In another set of experiments, the effect of the impact conditions on the energy release was investigated. The mass ratio of the $W$ was $45 \%$, and the thickness of the steel diaphragm was $2 \mathrm{~mm}, 3 \mathrm{~mm}, 4 \mathrm{~mm}$, and $5 \mathrm{~mm}$.

\section{Results and Discussion}

3.1. Mechanical Properties in Compression. Figure 11 shows an SEM micrograph of the fractures in the PTFE matrix. The main crack formed in the PTFE matrix is a fiber fracture, as shown by the arrow in the figure. It was formed because $\mathrm{Al}$ and $W$ particles rubbed against each other during the compaction process, absorbing most of the stress, and the energetic material was in the strain hardening stage. When the stress reached the compressive strength, the microcracks in the material further expanded and met, leading to the fracture of the energetic materials.

Figure 3 shows the quasistatic compressive stress-strain curve of the Al/PTFE/W energetic material. Table 2 shows the quasistatic mechanical properties of the Al/PTFE/W energetic materials in compression. Figure 4 shows the stress-strain curves of the Al/PTFE/W energetic materials with different $W$ contents (the strain rate was $4500 \mathrm{~s}^{-1}$ ). Figure 12 is the stress-strain curve of Al/PTFE/W energetic materials at different strain rates in the same ratio (the $W$ content was $45 \%)$. Table 3 shows the experimental scheme and dynamic mechanical properties of the $\mathrm{Al} /$ PTFE/W materials.

Figure 3 shows that, in the early stage of the curve, the material strain and stress were linearly elastic. When the strain continued to increase to a specific value, the rate of rise of the curve sharply decreased, showing a nonlinear state, and the material exhibited a yielding phenomenon.

Figure 3 and Table 2 show that the static yield strength and failure strength of the Al/PTFE/W energetic materials increased as the $W$ content increased because the $W$ particles had high strength and hardness and a body-centered cubic structure, which can be evenly distributed in the face-centered cubic unit cell of the aluminum particles, play a bonding role, and improve the yield strength of the composite material. In addition, as the $W$ content increased, the Al-PTFE binder phase could bond to additional $W$ particles, improving the contact area between the particles and increasing interfacial infiltration, which prevents crack propagation. Therefore, the failure strength of the composite 


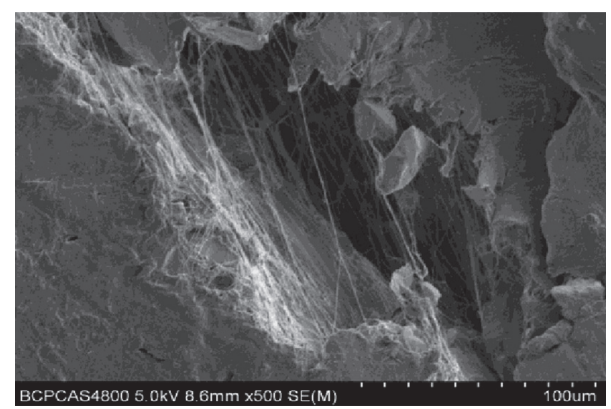

FIGURE 11: SEM micrograph of the fractures in the PTFE matrix.

TABLE 2: Quasistatic compressive mechanical properties of the Al/PTFE/W materials.

\begin{tabular}{lccc}
\hline Number & Mass ratio (W/Al/PTFE) $\%$ & Yield stress (MPa) & Failure stress (MPa) \\
\hline 1 & $0 / 26.5 / 73.5$ & 16.31 & 18.5 \\
2 & $25 / 19.9 / 55.1$ & 21.74 & 23.96 \\
3 & $45 / 14.6 / 40.4$ & 27.33 & 29.85 \\
4 & $60 / 10.6 / 29.4$ & 28.08 & 30.11 \\
\hline
\end{tabular}

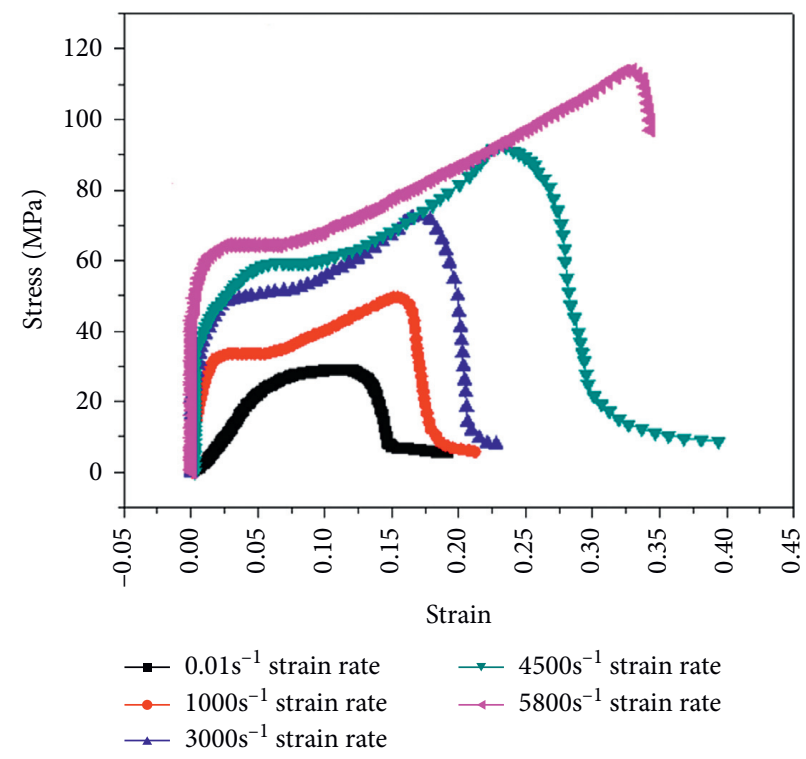

FIgURE 12: Stress-strain curves of Al/PTFE/W energetic materials at different strain rates with the same ratio (the $W$ content was $45 \%$ ).

TABle 3: Dynamic mechanical properties of the Al/PTFE/W materials.

\begin{tabular}{lcccc}
\hline Mass ratio of $W(\%)$ & Strain rate $\left(\mathrm{s}^{-1}\right)$ & Yield stress $(\mathrm{MPa})$ & Failure stress $(\mathrm{MPa})$ & Fracture strain \\
\hline 0 & 4500 & 46.3 & 66.28 & 74.91 \\
25 & 4500 & 52.89 & 88.36 & 0.238 \\
45 & 4500 & 58.25 & 92.58 & 0.225 \\
60 & 4500 & 59.78 & 29.85 & 0.187 \\
45 & 0.01 & 27.22 & 48.55 & 0.121 \\
45 & 1000 & 33.56 & 72.62 & 0.147 \\
45 & 3000 & 47.51 & 88.36 & 0.183 \\
45 & 4500 & 58.25 & 114.29 & 0.224 \\
45 & 5800 & 63.77 & & 0.325 \\
\hline
\end{tabular}


material was effectively improved. When the $W$ content was between $45 \%$ and $60 \%$, the adhesion of the Al-PTFE binder phase to the $W$ particles was basically saturated, and the increases in static yield strength and failure strength were limited.

Figure 4 and Table 3 show that the yield strength and compressive strength of the material increased gradually as the $W$ content in the composite increased under high strain rate conditions, which is similar to the trend at low strain rates. However, the strain at break decreased as the $W$ content increased, indicating that the addition of $W$ particles reduced the plasticity of the composite.

Figure 12 and Table 3 show that the yield strength, compressive strength, and strain at break of the Al/PTFE/W energetic materials were significantly improved under a high strain rate compared with the mechanical properties under static compression. The intensity increased as the strain rate increased, which had an obvious strain rate effect. In addition, the Al/PTFE/W energetic materials exhibited diminishing hardening properties after yielding under low strain rate conditions and exhibited incremental hardening properties under high strain rate conditions. Therefore, when the $\mathrm{Al} /$ PTFE/W energetic materials were subjected to impact loading, a secondary superposition of shock waves was formed inside the material, which helped to excite the activity of the Al/PTFE/ $\mathrm{W}$ material, which then reacted to release energy.

3.2. Impact Release Energy Characteristics. Due to the limitations of the experimental conditions, it was impossible to directly measure the energy released by the $\mathrm{Al} / \mathrm{Fe}$ energetic jet under impact loading. Therefore, it was necessary to establish a correlation between the measured overpressure signal and the energy release value. First, the pressure signal was divided into two stages: the rising stage and the releasing stage. The rising stage was equivalent to the sealed chamber environment. Thus, the relationship between the pressure value and energy value of the sealed chamber can be given by [16]

$$
\frac{\mathrm{d} Q}{\mathrm{~d} t}=\frac{V}{\gamma-1} \frac{\partial p}{\partial t}+\frac{\gamma p V}{m(\gamma-1)} \frac{\mathrm{d} m}{\mathrm{~d} t},
$$

where $Q$ is the increase in the internal energy of the closed chamber, $P$ is the pressure in the chamber, $V$ is the volume of the chamber, $m$ is the gas mass in the chamber, and $\gamma$ is the adiabatic index, for which a value of 1.4 was chosen.

Ames considered the rising stage of the overpressure in a chamber to take only a few milliseconds and the pressuretime relationship to be basically linear. Therefore, the presence of pressure relief holes can be neglected in the rising stage of the pressure curve. If the chamber is closed and the gas mass in the chamber is constant, equation (1) can be simplified as follows [16]:

$$
\Delta_{Q}=\frac{V}{\gamma-1} \Delta P .
$$

Equation (2) is the relationship between the peak value of the pressure in the container and the incremental change in the jet release energy during the rising stage of the pressure curve. When $P$ reaches the peak value of pressure, $Q$ is the maximum energy released during the rising stage of the pressure curve.

The main mechanism of the chemical reaction of metal under impact conditions involves the shockwave changing the internal structure and order of the material and causing a certain increase in the temperature [17], resulting in a hightemperature and high-pressure environment in which a local hot spot forms and a rapid diffusion reaction occurs. The reaction principle for energetic materials, such as explosives, is basically the same. Therefore, the impact energy level becomes a scale for judging whether a chemical reaction can be induced in the energetic material and the degree of reflection. Walker and Wasley proposed a method for calculating the shockwave energy, as shown in [18]

$$
\begin{aligned}
E & =P U_{P} t, \\
t & =\min \left\{\frac{2 r}{U_{S 1}}, \frac{2 h}{U_{S 2}}\right\}, \\
U_{P} & =\frac{-\left(\rho_{2} C_{2}+\rho_{1} C_{1}+2 \rho_{1} S_{1} V\right) \pm\left(\left(\rho_{2} C_{2}+\rho_{1} C_{1}+2 \rho_{1} S_{1} V\right)^{2}+4 \rho_{1}\left(\rho_{2} S_{2}-\rho_{1} S_{1}\right)\left(C_{1} V+S_{1} V^{2}\right)\right)^{1 / 2}}{2\left(\rho_{2} S_{2}-\rho_{1} S_{1}\right)},
\end{aligned}
$$

where $P$ is the pressure of the jet impact loading, $t$ is the pulse loading time, as shown in equation (4), $r$ is the radius of the jet when impacting a semi-infinite target, such as thick concrete, $h$ is the thickness of a limited target, such as a thin steel target (thinner than the jet diameter), and $U_{P}$ is the jet particle velocity, as shown in equation (5). The values of the impact parameters are shown in Table 4.

Figure 13 shows the original signal recorded by the sensor. There was a brief pulse jump in the initial phase of the signal when the charge was ignited, and then a short pulse peak was generated. This was due to the detonation wave formed by the detonation of the charge propagating faster in the air than in the jet, and the shock pulse was generated before the jet reached the test container. The two pulse signals mentioned above should be eliminated in the analysis of the jet energy release. The rapid rise of the detonation pulse and slow decrease of the peak value indicate that the exothermic chemical reaction induced by the 
TABLE 4: Values of the impact parameters.

\begin{tabular}{lcccccccc}
\hline$\rho_{1}^{a}\left(\mathrm{~kg} / \mathrm{m}^{3}\right)$ & $\rho_{2}^{b}\left(\mathrm{~kg} / \mathrm{m}^{3}\right)$ & $C_{1}^{c}(\mathrm{~m} / \mathrm{s})$ & $C_{2}^{d}(\mathrm{~m} / \mathrm{s})$ & $S_{1}^{e}$ & $S_{2}^{f}$ & $V^{g}(\mathrm{~m} / \mathrm{s})$ & $U_{P}(\mathrm{~m} / \mathrm{s})$ & $U_{S 2}^{h}(\mathrm{~m} / \mathrm{s})$ \\
\hline 3750 & 7850 & 3334 & 4569 & 1.44 & 1.49 & 3835 & 1300 & 6506 \\
\hline
\end{tabular}

${ }^{a}$ The density of the jet. ${ }^{b}$ The density of the target. ${ }^{\mathrm{c}}$ The acoustic velocity of the jet. ${ }^{\mathrm{d}}$ The acoustic velocity of the target. ${ }^{\mathrm{e}}$ The empirical parameters of the jet. ${ }^{\mathrm{f}}$ The empirical parameters of the target. ${ }^{g}$ The velocity of the jet. ${ }^{\mathrm{h}}$ The shock wave velocities of the target.

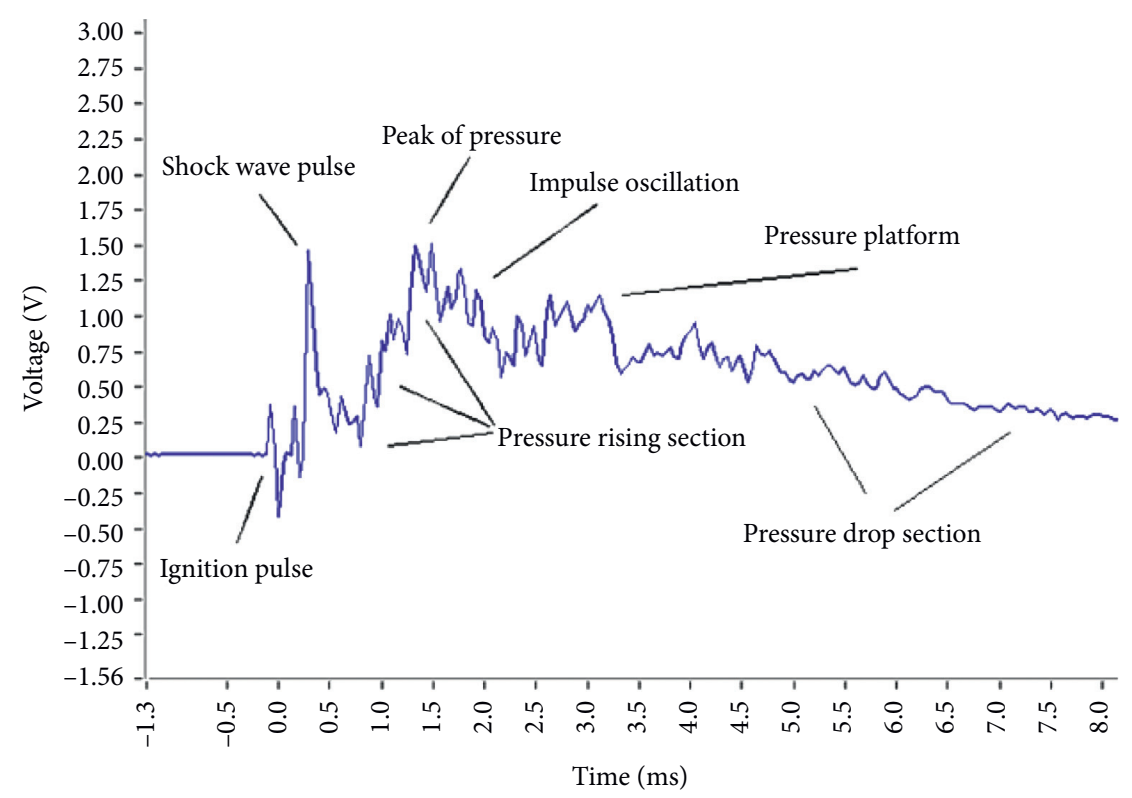

FIGURE 13: The raw signal recorded by the sensor.

energetic jet under the impact produced a pressure. There was a short platform as the pulse decreased, which indicates that the secondary reaction of the residual substance of the energetic jet occurred after the impact, and the released energy was in balance with the dissipated energy. The duration of the platform can also be used to indicate if the energetic material reacted adequately. Regular waveform oscillations occurred during the rise and fall of the voltage signal. This was due to a small sloshing of the container and the reflection of the shock wave in the container wall under the high-speed impact of the jet. The data needed to be filtered and smoothed. The following content was analyzed after smoothing and filtering the pressure-time curve unless otherwise specified.

Figure 14 shows the energy release phenomenon of impacting the $4 \mathrm{~mm}$ steel plate with the Al/PTFE/W jets with $45 \% W$ content. The intense chemical reaction occurred after the jet impacted the steel target, accompanied by luminescence and heating. There was a clear jet of sparks in the pressure relief hole, and the reaction reached the most violent state at $25 \mathrm{~ms}$. Figure 15 shows the pressure-time curve generated when the Al/PTFE/W energetic jets with different $W$ contents impacted the $4 \mathrm{~mm}$ diaphragm. Figure 16 shows the pressure-time curve generated when the Al/PTFE/W energetic jets with a mass content of $45 \% W$ hit different target thicknesses.

Figure 17 compares the pressure peak and energy release values of the Al/PTFE/W energetic jets with different $W$ contents. Figure 18 compares the platform time and pressure duration of the Al/PTFE/W energetic jets with different $W$ contents. Figure 19 compares the pressure peak and energy release of the Al/PTFE/W energetic jets for different target thicknesses. Figure 20 compares the platform time and pressure duration of the Al/PTFE/W energetic jets for different target thicknesses.

Figures 15 and 17 and Table 2 show that, as the $W$ content in the energetic jet increased, the pressure peak and energy release values of the jets impacting the $4 \mathrm{~mm}$ thick target plate first increased and then decreased. When the $W$ content was $45 \%$, the pressure peak and total release energy of the Al/PTFE/W energetic jet were the highest. When the $W$ content was less than $45 \%$, the intensity of the Al/PTFE/ $\mathrm{W}$ energetic jet was relatively low, and some materials broke and scattered after being subjected to impact loading, which did not have a subsequent reaction. When the $W$ content reached $60 \%$, the content of the Al/PTFE capable of a chemical reaction was low, thereby reducing the efficiency of the reaction release energy.

Figures 16 and 18 and Table 3 show that when the $W$ content was $45 \%$, the pressure platform time and pressure duration of the Al/PTFE/W energetic jets were also the longest, indicating that the $\mathrm{Al} / \mathrm{PTFE} / \mathrm{W}$ energetic jets with a $W$ content of $45 \%$ had the longest reaction time and the highest energy release efficiency after impacting the target.

Figures 16 and 19 and Table 3 show that, as the thickness of the target increased, that is, as the impact energy of the $\mathrm{Al} /$ 


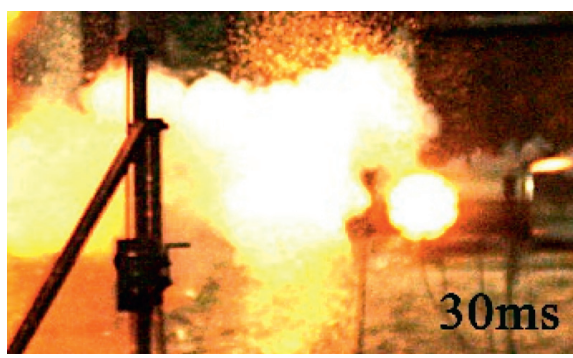

(a)

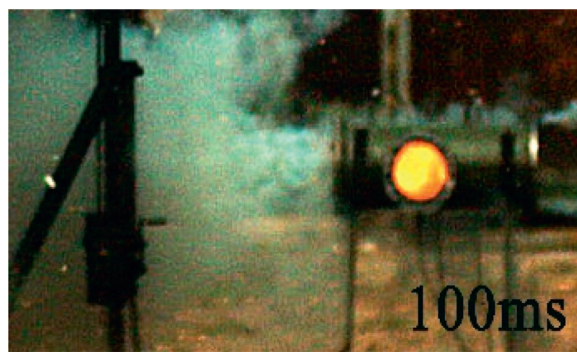

(c)

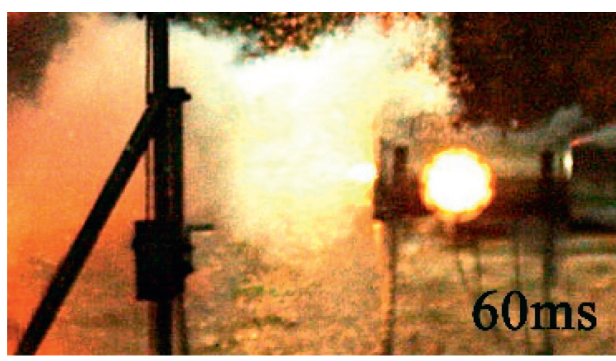

(b)

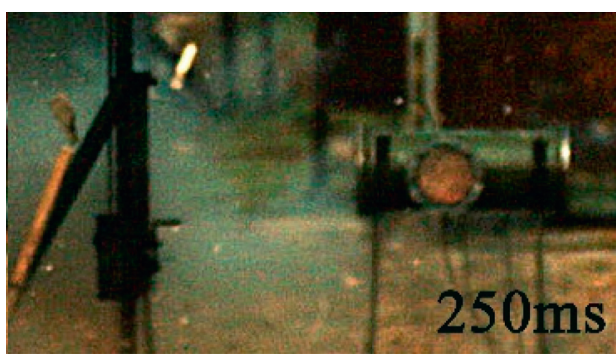

(d)

FIgURE 14: Energy release phenomenon of impacting a $4 \mathrm{~mm}$ steel plate with the Al/PTFE/W jets with a $45 \% W$ content.

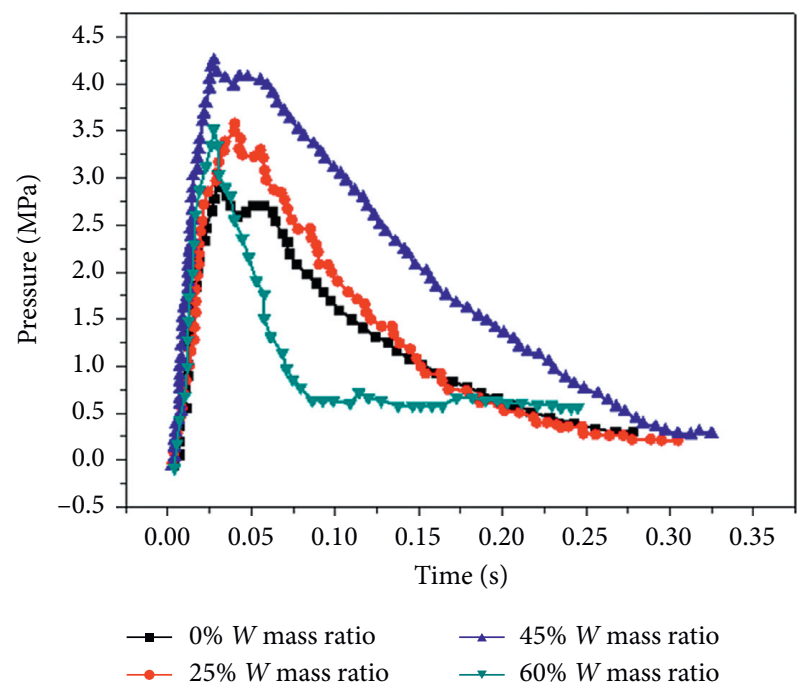

FIGURE 15: Comparison of the pressure-time curves of the $\mathrm{Al} /$ PTFE/W energetic jets with different $W$ contents.

PTFE/W energetic jets increased, the pressure peak and energy release values increased. When the thickness of the target plate was $4 \mathrm{~mm}$ and $5 \mathrm{~mm}$, the energy generated by the $\mathrm{Al} / \mathrm{PTFE} / \mathrm{W}$ energetic jets was almost the same. This result shows that when the impact energy was $106.1 \mathrm{MJ} / \mathrm{m}^{2}$, the chemical reaction of the Al/PTFE/W (45\%) energetic jets was saturated.

Figures 16 and 20 show that the secondary reaction time and the total energy release time of the Al/PTFE/W energetic jets impacting different thickness targets varied greatly. When the jet impacted a $2 \mathrm{~mm}$ target (the impact energy was $53.1 \mathrm{MJ} / \mathrm{m}^{2}$ ), the secondary reaction time of the jet residual

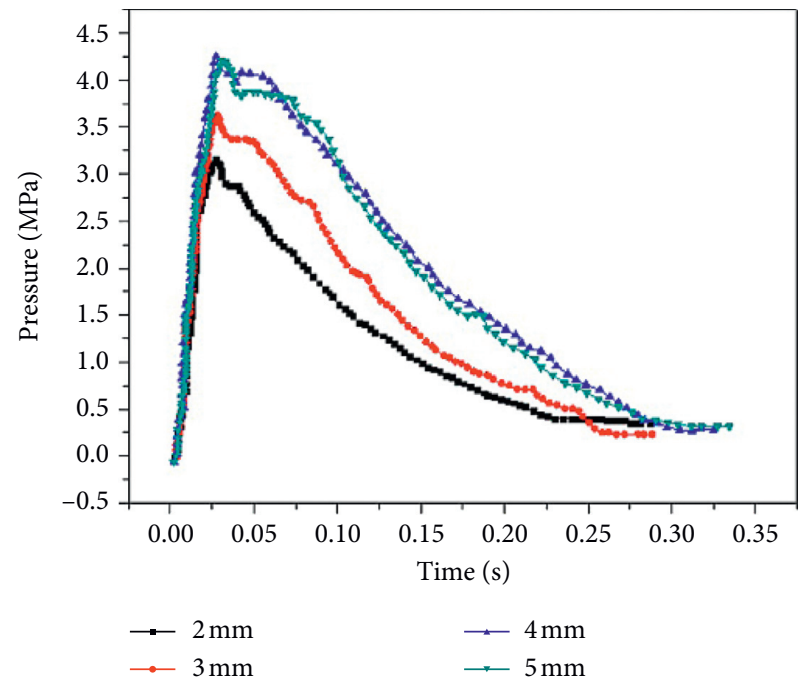

FIGURE 16: Comparison of the pressure-time curves of the target plates with different thicknesses.

material was very short at only $5.6 \mathrm{~ms}$. In contrast, when the jet impacted a $4 \mathrm{~mm}$ target (the impact energy was $106.1 \mathrm{MJ} /$ $\mathrm{m}^{2}$ ), the secondary reaction time was $47.5 \mathrm{~ms}$. When the impact energy continued to increase, the secondary reaction time and the total energy release time decreased. This indicates that the excessive impact energy caused the jet to dissipate to an elevated degree when it hit the target, resulting in a decrease in the energetic materials that can induce chemical reactions.

In summary, the results of these experiments demonstrate that the energy release of the Al/PTFE/W energetic jets under loading was influenced by the $W$ content and impact energy. 


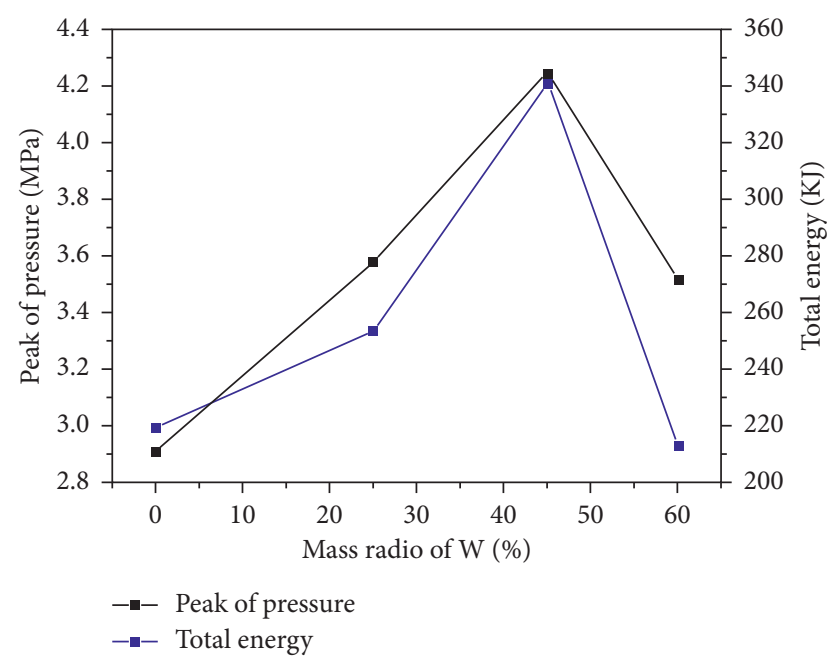

FIGURE 17: Comparison of the overpressure peak value and energy release value of energetic jets with different $W$ contents.

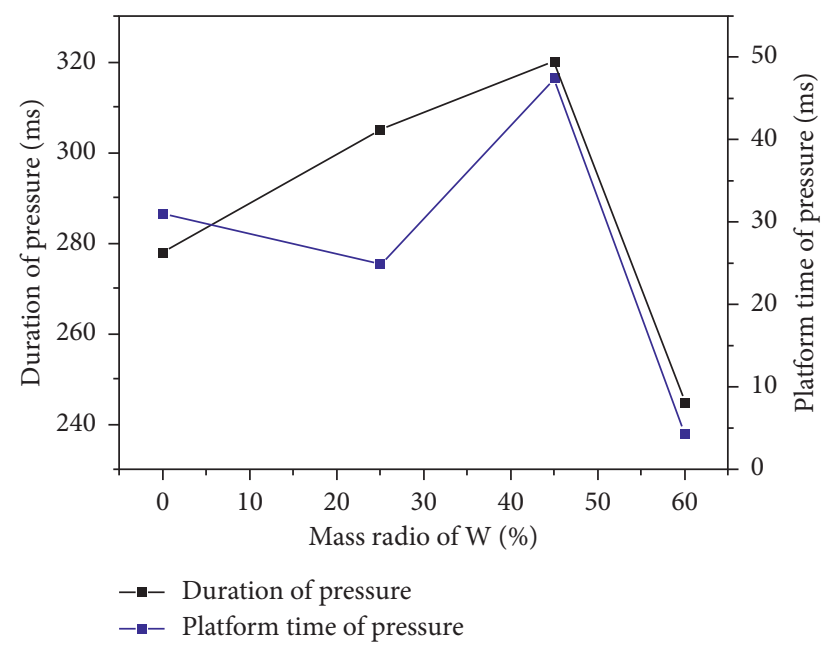

FIGURE 18: Comparison of the platform time and pressure duration for the Al/PTFE/W energetic jets with different $W$ contents.

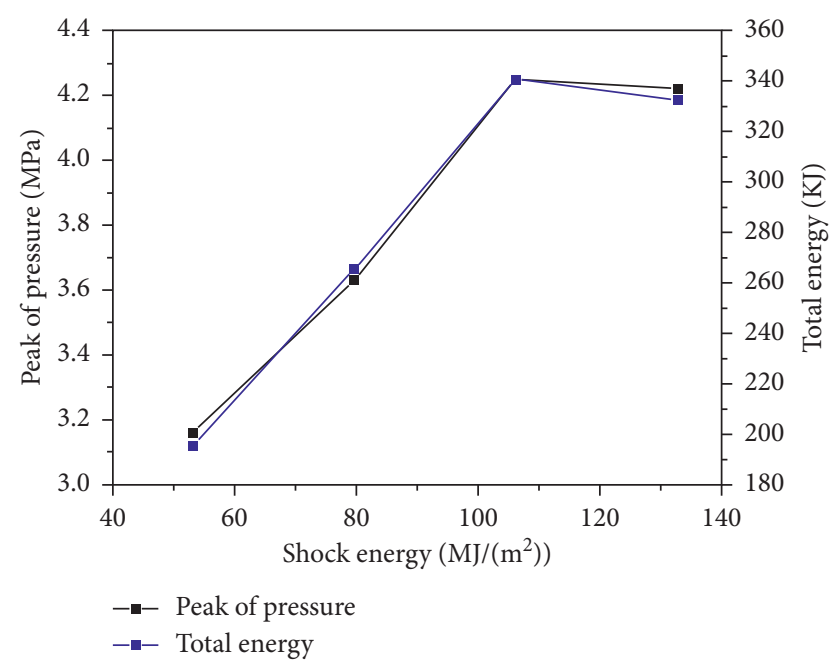

FIGURE 19: Comparison of the peak pressure and energy release of the Al/PTFE/W energetic jets for different target thicknesses.

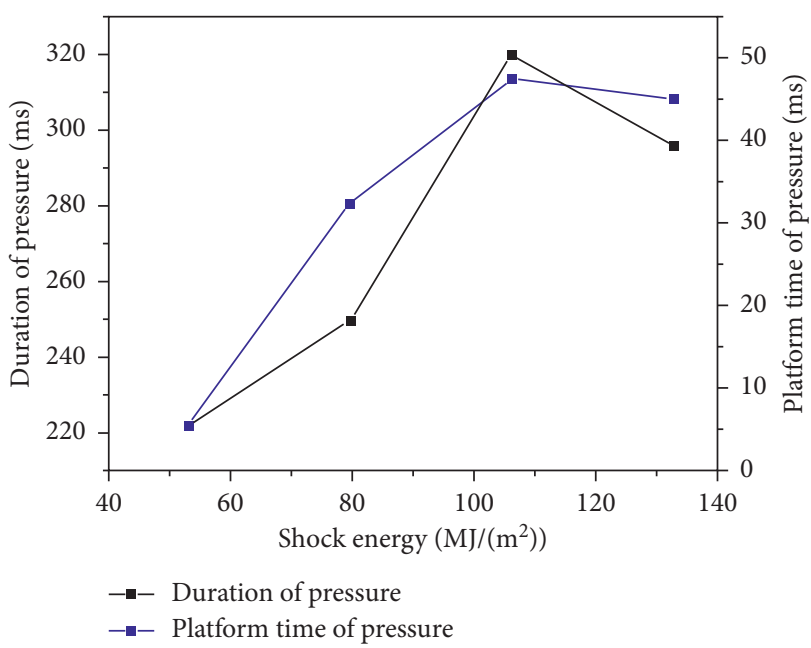

FIGURE 20: Comparison of platform time and pressure duration for the Al/PTFE/W energetic jets for different target thicknesses.

\section{Conclusions}

The main contribution of this paper is a study of the mechanical properties and energy release characteristics of $\mathrm{Al} /$ PTFE/W energetic materials. The influence of different material ratios and impact conditions on the energy release characteristics of $\mathrm{Al} / \mathrm{PTFE} / \mathrm{W}$ energetic jets was obtained by constructing a dynamic energy harvesting test system. The following conclusions are drawn from the study:

(1) The static yield strength and failure strength of the $\mathrm{Al} / \mathrm{PTFE} / \mathrm{W}$ composites increase as the $W$ content increases. When the $W$ content is between $45 \%$ and $60 \%$, the adhesion of the Al-PTFE binder phase to the $W$ particles is substantially saturated, and the increases in the static yield strength and failure strength of the composites are limited.

(2) At high strain rates, the Al/PTFE/W composites have a significant strain rate effect. As the $W$ content in the composite increases, the yield strength and compressive strength of the material increase gradually, but the strain at break decreases as the $W$ content increases. In addition, the $\mathrm{Al} / \mathrm{PTFE} / \mathrm{W}$ energetic materials exhibit diminishing hardening properties after yielding under low strain rate conditions and increasing hardening properties under high strain rate conditions.

(3) When the $W$ content is $45 \%$, the Al/PTFE/W energetic energy jet pressure peak, total release energy, pressure platform duration, and total pressure duration are the highest. As the impact energy increases, the pressure peak and energy release values of the energetic jets increase. When the impact energy reaches $106.1 \mathrm{MJ} / \mathrm{m}^{2}$, it induces a sufficient chemical reaction and the longest secondary reaction time of the Al/PTFE/W (45\%) energetic jet.

The results show that PTFE material can be applied to jets and release energy under impact loading, which provides 
a theoretical and experimental basis for the application of high-energy jets. The next step will focus on determining if, compared with the inert jet, the PTFE jet has a damage enhancement effect when penetrating the target.

\section{Data Availability}

The raw/processed data required to reproduce these findings cannot be shared at this time due to legal and ethical reasons.

\section{Conflicts of Interest}

The authors declare that there are no conflicts of interest regarding the publication of this paper.

\section{Acknowledgments}

The authors thank Qiang Li from the North University of China for providing them with the impact energy releasing test instrument.

\section{References}

[1] X. Fan, G. Li, Y. Guo et al., "Role of reinforcement types and silica nanoparticles on tribofilm growth at PTFE-Steel interface," Tribology International, vol. 143, Article ID 106035, 2020.

[2] E. C. Koch, Metal-Fluorocarbon Based Energetic Materials, Wiley-VCH Verlag Gmbh \& Co. KGa A, Weinheim, Germany, 2012.

[3] B. Feng, Y.-C. Li, S.-Z. Wu, S.-z. Wu, Z.-M. Tao, and X. Fang, "A crack-induced initiation mechanism of Al-PTFE under quasi-static compression and the investigation of influencing factors," Materials \& Design, vol. 108, pp. 411-417, 2016.

[4] R. G. Ames, "Energy release characteristics of impact-initiated energetic materials," MRS Proceedings, vol. 18, pp. 21-24, 2006.

[5] W. J. Mock and J. T. Drotar, "Effect of aluminum particle size on the impact initiation of pressed PTFE/Al composite rods," Shock Compression of Condensed Matter, vol. 955, pp. 971974, 2007.

[6] J.-F. Shuai, J.-W. Jiang, S.-Y. Wang, J.-B. Men, and C.-Y. Xie, "Compound reactive fragment penetrating steel target," Chinese Journal of Energetic Materials, vol. 17, pp. 722-725, 2009.

[7] C.-Y. Xie, J.-W. Jiang, J.-F. Shuai, J.-B. Men, and S.-Y. Wang, "Experimental study on the damage effect of compound reactive fragment penetrating diesel oil tank," Chinese Journal of High Pressure Physics, vol. 23, pp. 447-452, 2009.

[8] C.-L. Xin, W.-Q. Shi, L.-L. Zhang, X.-W. Long, G.-J. Liu, and J.-S. Wang, "Damage effects of shaped charge comprised of reactive materials liner to the steel target," in Proceedings of the 6th International Symposium on Energetic Materials and Insensitive Ammunition, China Academy of Engineering Physics, Sendai, Japan, November 2014.

[9] H.-F. Wang, Y.-F. Zheng, Q.-B. Yu, Z.-W. Liu, and W.-M. Yu, "Experimental research on igniting the aviation kerosene by reactive fragment," Acta Armamentarii, vol. 33, pp. 11481152, 2012.

[10] H. Wang, Y. Zheng, Q. Yu, Z. Liu, and W. Yu, "Impact-induced initiation and energy release behavior of reactive materials," Journal of Applied Physics, vol. 110, no. 7, Article ID 074904, 2011.
[11] X. F. Zhang, A. S. Shi, L. Qiao, J. Zhang, Y. G. Zhang, and Z. W. Guan, "Experimental study on impact-initiated characters of multifunctional energetic structural materials," Journal of Applied Physics, vol. 113, 2013.

[12] P. Luo, Z. Wang, C. Jiang, L. Mao, and Q. Li, "Experimental study on impact-initiated characters of $\mathrm{W} / \mathrm{Zr}$ Energetic Fragments," Materials \& Design, vol. 84, pp. 72-78, 2015.

[13] L. Wang, J. Liu, S. Li, and X. Zhang, "Investigation on reaction energy, mechanical behavior and impact insensitivity of W-PTFE-Al composites with different $W$ percentage," $M a$ terials \& Design, vol. 92, pp. 397-404, 2016.

[14] X.-B. Zhang, J.-X. Lin, L. Wang et al., "Effects of $\mathrm{Al}$ and $W$ particle size on combustion characteristics and dynamic response of W-PTFE-Al composites," Rare Metal Materials and Engineering, vol. 47, pp. 1723-1728, 2018.

[15] M. R. Khosravani, P. Wagner, D. Fröhlich, and K. Weinberg, "Dynamic fracture investigations of ultra-high performance concrete by spalling tests," Engineering Structures, vol. 201, Article ID 109844, 2019.

[16] R. G. Ames, "Vented chamber calorimetry for impact-initiated energetic materials," in Proceedings of the 43rd AIAA Aerospace Sciences Meeting and Exhibit, AIAA, Reno, Nevada, pp. 10-13, January 2005.

[17] X.-F. Zhang, X.-N. Zhao, and L. Qiao, “Theoretical analysis of impact reactive metal," Explosion, and Shock Waves, vol. 30, pp. $145-151,2010$.

[18] F. E. Walker and R. J. Wasley, "Critical energy for shock initiation of heterogeneous explosives," Explosive Stoffe, vol. 17 , no. $1,1969$. 